\title{
3-D Modeling of Triple Junction Solar Cells on 2-D Gratings with Optimized Intermediate and Back Reflectors
}

\author{
O. Isabella, M. El-Shinawy, S. Solntsev, M. Zeman \\ Photovoltaic Materials and Devices/Dimes, Delft University of Technology, Feldmannweg 17, 2628 CT Delft, \\ e-mail: olindo.isabella@gmail.com
}

\begin{abstract}
Superstrate thin-film silicon triple-junction solar cells on 2-D gratings were optimized using opto-electrical modeling. Tuning the thickness of intermediate and back reflectors and the band gap of the middle cell resulted in $17 \%$ initial efficiency.

OCIS codes: $050.1755,050.1950,310.6845$
\end{abstract}

Light trapping in thin-film silicon solar cells is accomplished by light scattering at internal interfaces and reflection at the rear side. We introduced glass-based 2-D pyramidal gratings $(P=1500 \mathrm{~nm})$ for light scattering at the front side of a superstrate triple-junction solar cell structure and a $\mathrm{ZnO} / \mathrm{Ag}$ stack for reflection at the back side. We optimized both i) a thin design for a high stability and throughput (target: $J_{P H-T O T}=27.9 \mathrm{~mA} / \mathrm{cm}^{2}$ ) and ii) thick design for a high efficiency (target: $J_{P H-T O T}=30 \mathrm{~mA} / \mathrm{cm}^{2}$ ). The investigated triple junction solar cells had the following structure: glass-based textured substrate coated with a thin film of $\operatorname{In}_{2} \mathrm{O}_{3}: \mathrm{H}(\mathrm{IOH})$ [2] on which three $p$ - $i-n$ junctions were stacked on top of each other with a-Si:H, a-SiGe:H, and nc-Si:H absorber layers, respectively. The rear side was completed with $\mathrm{ZnO} / \mathrm{Ag}$ reflector/electrode. $\mathrm{SiO}_{\mathrm{x}}$ films $\left(\mathrm{n}_{\mathrm{SiOx}}=2.1\right.$ at $\left.770 \mathrm{~nm}\right)$ were used as n-type layer for the top and middle component cells and as intermediate reflectors (IR1 and IR2, respectively) at the same time. Optical simulations were carried out with 3D finite element method package (HFSS) [1]. The calculated optical generation rate was used as input for optoelectrical simulations carried out by the ASA software.

We carried out optimization of the current-matched solar cell structure in order to achieve a higher $J_{P H-T O T}$ with respect to the specified target. The results of the optimization are reported in Fig. 1. We tuned (i) the thickness of intermediate and back reflectors (series 1-3), (ii) the thickness of front IOH and top p-layer (series 4), and (iii) the pyramid shape (series 5-8). In order to obtain current-matching after changing the band gap of a-SiGe:H absorber layer, we varied the thickness of absorber layers (series 9 and 10). We found two structures (denoted \#2 and \#3) fulfilling our requirements and their details are reported in Fig. 1. We show in Fig. 2 that using our light trapping scheme in a single junction nc-Si:H solar cell ( $\left.\mathrm{i}_{\mathrm{nc}-\mathrm{Si}: \mathrm{H}}=2.8 \mu \mathrm{m}\right)$ the absorption limit predicted by $4 \mathrm{n}^{2}$ enhancement using ideal scattering is approached. In Fig. 3 the spectral absorption rate profile in the triple-junction cell is shown. From these absorption rate profiles the optical generation rates were calculated and used in electrical simulations. Modeling enables us to visualize the spatial distribution of the magnitude of electric field inside the complete cell at different wavelengths that helps us to study the contribution of the textured back contact to scattering (see Fig. 4). Analysis of the total reflectance and absorptance in absorber and supporting layers guides us in further optimization of triple junction devices (see Fig. 5).

To evaluate external parameters from electrical simulations we used structures \#2 and \#3 in both current-matched and current-mismatched conditions. The latter was achieved by using not-optimized thicknesses for IR's and back ZnO. The fill factor was found to be higher for thinner and mismatched structures, while the open circuit voltage resulted slightly higher only for thinner structures. An interplay between short circuit current density and fill factor occurred in case of structures \#3, for which the current-mismatched structure presented slightly higher efficiency. Current matched thin (thick) design resulted in $16.3 \%(17.1 \%)$ potential initial efficiency.

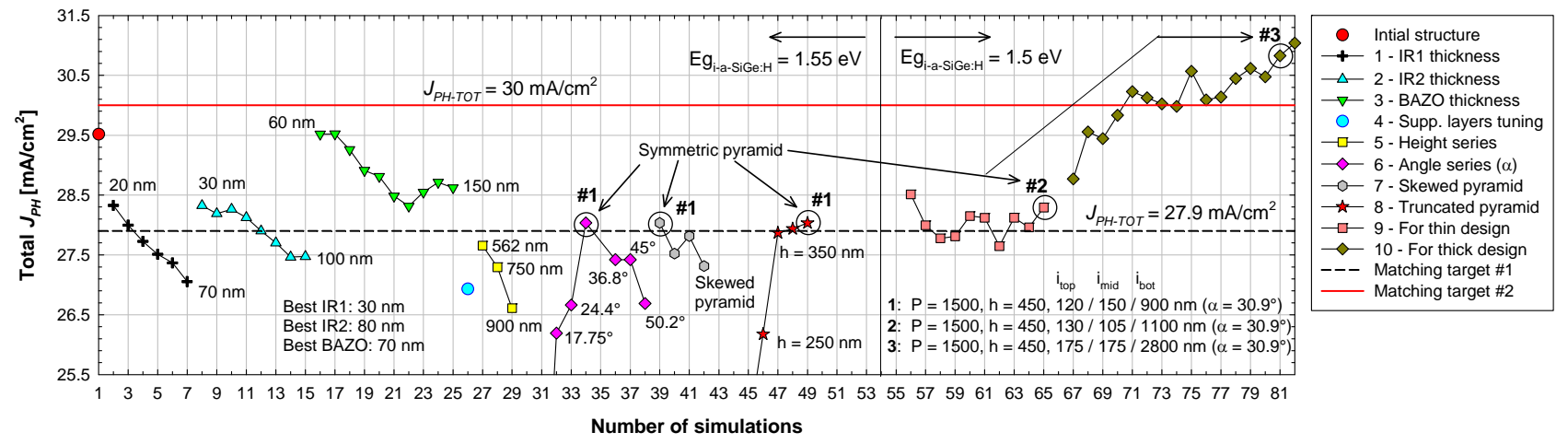

Fig. 1 Total photo-current density in simulated triple junction cells. From left to right results of the subsequent optimization series are reported. Circled dots indicate current-matched triple junction devices above at least one of the two targets. 


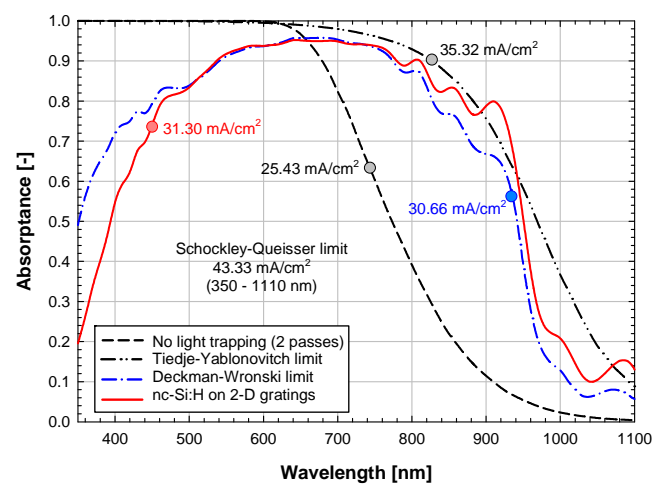

Fig. 2 Absorption limits and absorptance of nc-Si:H single junction solar cell $\left(\mathrm{i}_{\mathrm{bottom}}=2.8 \mu \mathrm{m}\right)$ related to thick design \#3.

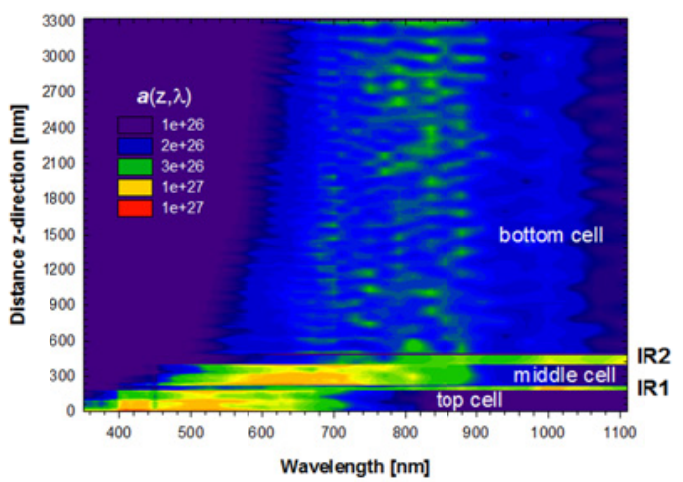

Fig. 3 Spectral absorption rate profile related to thick design \#3 (from bottom to top: top cell / IR1 / middle cell / IR2 / bottom cell).
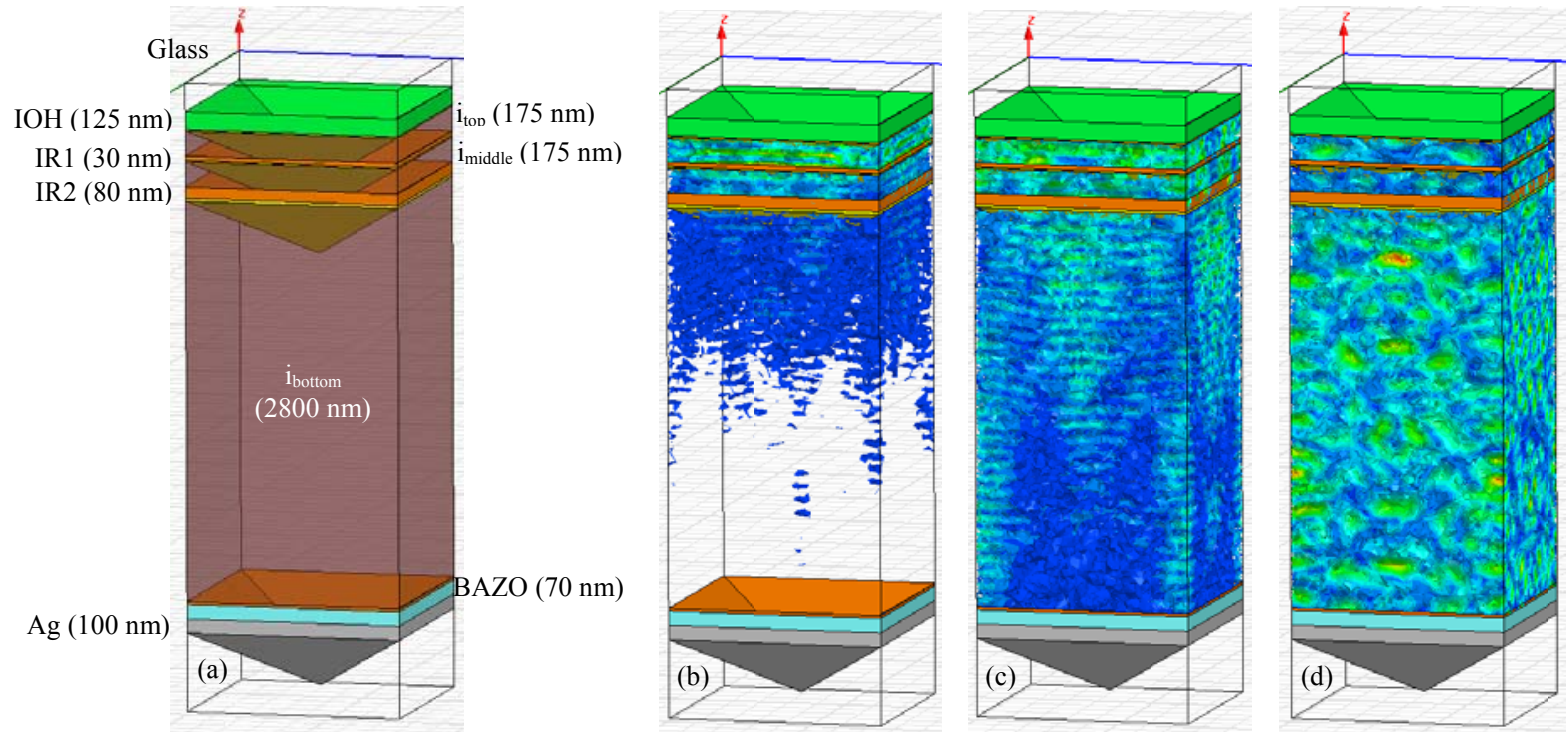

Fig. 4 (a) Structure of thick design \#3 and electric field magnitude spatial distribution at $610 \mathrm{~nm}$ (b), $680 \mathrm{~nm}$ (c) and $833 \mathrm{~nm}$ (d).

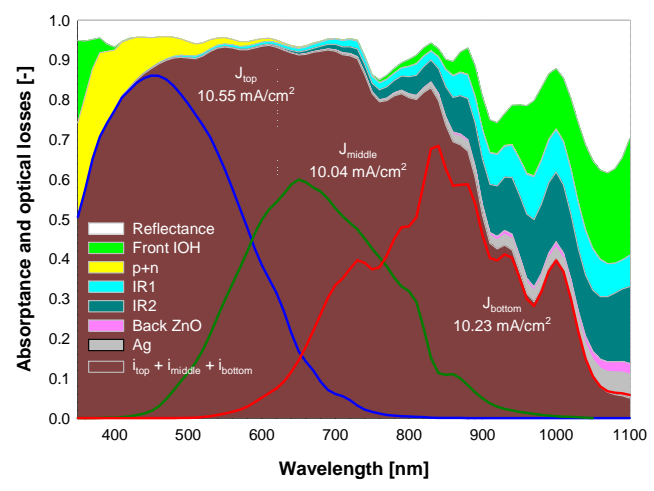

Fig. 5 Absorptance and optical losses of the thick design \#3. Implied photocurrent density of component cells equals $10 \mathrm{~mA} / \mathrm{cm}^{2}$.

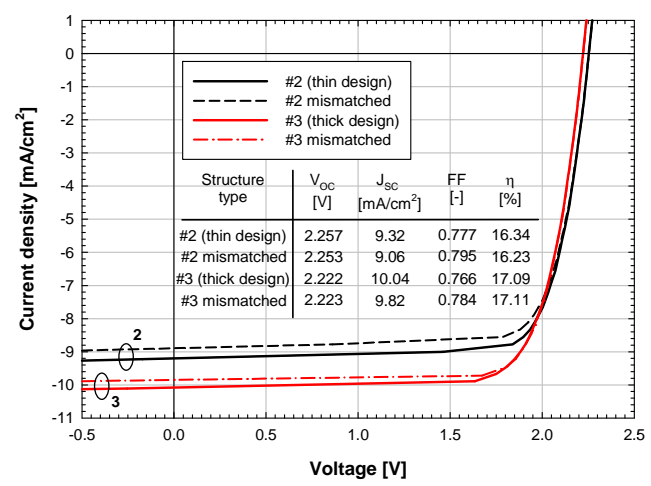

Fig. $6 \mathrm{~J}-\mathrm{V}$ characteristics of matched / mismatched structures \#2 and \#3. In the inset simulated initial external parameters are reported.

\section{References}

[1] O. Isabella, S. Solntsev, D. Caratelli, M. Zeman, “3-D optical modeling of thin-film silicon solar cell on diffraction gratings," Progress Photovoltaics: Research and Applications 2012; DOI: 10.1002/pip.1257.

[2] T. Koida, H. Fujiwara, M. Kondo, "Reduction of Optical Loss in Hydrogenated Amorphous Silicon/Crystalline Silicon Heterojunction Solar Cells by High-Mobility Hydrogen-Doped In2O3 Transparent Conductive Oxide," Applied Physics Express 2008; 1: 041501, DOI 10.1143/APEX.1.041501. 